\title{
Effects Induced by the Initial Condition in the Quantum Kibble-Zurek Scaling for Changing the Symmetry-Breaking Field
}

\author{
Liang-Jun Zhai ${ }^{1, *}$ and Shuai Yin ${ }^{2, *}$ \\ 1 School of Mathematics and Physics, Jiangsu University of Technology, Changzhou 213001, China \\ 2 Department of Physics, National Tsing Hua University, Hsinchu 30013, Taiwan \\ * Correspondence: zhailiangjun@jsut.edu.cn (L.-J.Z.); sysuyinshuai@gmail.com (S.Y.); \\ Tel.: +86-519-8695-3419 (L.-J.Z.); +86-10-6278-9589 (S.Y.)
}

Academic Editor: Ignazio Licata

Received: 18 November 2016; Accepted: 9 December 2016; Published: 14 December 2016

\begin{abstract}
The Kibble-Zurek scaling describes the driven critical dynamics starting with an equilibrium state far away from the critical point. Recently, it has been shown that scaling behaviors also exist when the fluctuation term changes starting near the critical point. In this case, the relevant initial conditions should be included in the scaling theory as additional scaling variables. Here, we study the driven quantum critical dynamics in which a symmetry-breaking field is linearly changed starting from the vicinity of the critical point. We find that, similar to the case of changing the fluctuation term, scaling behaviors in the driven dynamics can be described by the Kibble-Zurek scaling with the initial symmetry-breaking field being included as its additional scaling variable. Both the cases of zero and finite temperatures are considered, and the scaling forms of the order parameter and the entanglement entropy are obtained. We numerically verify the scaling theory by taking the quantum Ising model as an example.
\end{abstract}

Keywords: Kibble-Zurek scaling; initial condition; symmetry-breaking field; finite temperature

\section{Introduction}

Recent experimental progress made in cold-atom systems has led to many studies on the nonequilibrium behaviors in quantum many-body systems [1-3]. Among them, the Kibble-Zurek mechanism, which was first proposed by Kibble in cosmology [4], and then by Zurek in condensed matter physics [5], has been generalized to describe the driven quantum critical dynamics starting with a state far away from the critical point [6-23]. According to the Kibble-Zurek mechanism [1-3], the whole driven process can be divided into three stages: one impulse stage sandwiched by two adiabatic stages. At the first stage, the energy gap is very large, the excitation induced by the external driving can be ignored, and thus the system evolves adiabatically along the equilibrium state; then, when the system is near its critical point, the gap becomes smaller and cannot suppress the excitations induced by the external driving, and the system is in an impulse stage in which the external driving dominates. After this stage, the system enters another adiabatic stage. At the boundary between this latter adiabatic stage and the impulse stage, the Kibble-Zurek mechanism shows that the number of topological defects can be scaled by the driving rate [1-3]. Recently, the Kibble-Zurek mechanism has been verified experimentally in trapped-ion systems and Bose-Einstein condensates [24-27]. Furthermore, the full scaling forms have been proposed to explain the scaling behaviors in the whole process [18-23,28,29]. Additionally, these scaling forms have been employed to numerically estimate the critical point and critical exponents in both classical and quantum phase transitions [28-32].

The original Kibble-Zurek mechanism requires an initial adiabatic stage before the impulse stage [6-23]. Since the relaxation time in this initial stage is very small, effects induced by the initial 
condition will be smeared by the fluctuation in a microscopic time scale in the following driven process. So, the original Kibble-Zurek mechanism shows that the scaling of physical quantities is independent of the detailed information of the initial condition.

However, when the driven dynamics are started up in the vicinity of the critical point, the initial effects will be remembered for a macroscopic time scale because the relaxation time near the critical point is very large. As a result, the initial condition will affect the driven dynamics. This inspires many studies on how to generalize the Kibble-Zurek mechanism to take the effects induced by the initial conditions into account in both classical and quantum phase transitions [33-38].

Since thermal effects cannot be perfectly excluded in real experiments, the study on the quantum critical dynamics at finite temperatures [35-39] is another important issue. For the driven dynamics in a closed system, the thermal effects can only be involved in the initial condition [35-38]. It has been demonstrated that for a closed system, the thermal effects involved in the driven critical dynamics are closely related to the initial distance to the critical point [38].

In contrast to previous studies, in which the fluctuation term is changed from the vicinity of the critical point [35-38], here we study the quantum driven critical dynamics by changing a symmetry-breaking field starting with an equilibrium state near the critical point. We will show that the scaling behaviors also exist. Similar to the case of changing the fluctuation term, the initial symmetry-breaking field should be included in the full scaling form as its additional scaling variable. The general scaling forms will be obtained for both the zero temperature and finite temperature cases. In particular, we will consider the scaling forms of the order parameter and the entanglement entropy. The scaling theory will be verified in the one-dimensional (1D) quantum Ising model.

The rest of the paper is organized as follows. In Section 2, we briefly review the Kibble-Zurek scaling (KZS) and its generalization. Then, in Section 3, we show the scaling theory of driven critical dynamics for changing the symmetry-breaking field starting near the critical point. Section 4 will introduce the 1D quantum Ising model and the numerical method. Then, the numerical results to verify the scaling theory are shown in Section 5. Finally, we will give a summary in Section 6.

\section{Kibble-Zurek Scaling and Its Generalization}

Here we give a brief review on the KZS. The original KZS often considers the case of changing the fluctuation field starting from a state far from the critical point and only focuses on the scaling of the number of topological defects at the boundary between the impulse and adiabatic region, while the dynamics in the impulse region are assumed to be frozen [1-17]. However, this is apparently not the real situation. Finite-time scaling (FTS) $[28,29]$ provides a theory to understand the dynamics in the impulse region and build a full scaling theory of the KZS. The FTS shows that an effective time scale, which is induced by the external driving, dominates the dynamics in the impulse region, since in this region this effective time scale is the shortest time scale compared with other macroscopic time scales, such as the reaction time scale of the system. This is similar to the finite-size scaling theory, which states that the lattice size dominates the critical behavior when it is smaller than the correlation length.

We start from the usual finite-size scaling theory. Near the critical point, the scale transformation of the order parameter $M$ reads as [39]

$$
M(g, L)=b^{-\beta / v} M\left(g b^{1 / v}, L b^{-1}\right)
$$

in which $g$ is the distance of the fluctuation term to the critical point and $L$ is the lattice size, $\beta$ is defined as $M \sim(-g)^{\beta}$ for $g<0$ [39], and $v$ is defined as $\xi \sim|g|^{-v}$ with $\xi$ being the correlation length. The finite-size scaling theory shows that when $L \ll \xi$, the scaling behavior is dominated by $L$. Accordingly, $M$ should satisfy the finite-size scaling form [39].

$$
M(g, L)=L^{-\beta / v} f_{1}\left(g L^{1 / v}\right)
$$


in which $f_{1}$ is an analytic scaling function and the effect induced by $g$ is only a perturbation. In contrast, when $L \gg \xi$, $M$ satisfies

$$
M(g, L)=g^{\beta} f_{2}\left(L^{-1} g^{-v}\right)
$$

In this case, $g$ dominates the scaling behavior and $L$ is a perturbation.

Similar to the finite-size scaling theory, the FTS theory explains the driven dynamic scaling behavior by noting that the external driving will introduce an effective time scale $\zeta_{e}$, which competes against the relaxation time $\zeta_{s} \sim \xi^{z}[28,29]$, in which $z$ is the dynamic. To be explicit, we consider the case for changing the fluctuation term $g$ as $g=R_{g} t+g_{0}$ with $g_{0}$ being far from the critical point. In this case, the driving-induced effective time scale is $\zeta_{e} \sim R_{g}{ }^{-z / r_{g}}$, in which $r_{g}=z+1 / \nu$. Similar to Equation (1), the evolution of the order parameter $M$ under the driving should satisfy

$$
M\left(g, R_{g}\right)=b^{-\beta / v} M\left(g b^{1 / v}, R_{g} b^{r_{g}}\right)
$$

In the FTS region, in which $\zeta_{e} \ll \zeta_{s}$, $\zeta_{e}$ controls the dynamics and the external driving dominates. By setting $R_{g} b^{r_{g}}=1$ in Equation (1), one can obtain the scaling form of $M$,

$$
M\left(g, R_{g}\right)=R_{g}{ }^{\beta / v r_{g}} f_{3}\left(g R_{g}{ }^{-1 / v r_{g}}\right)
$$

Compared with the original KZS, one finds that the FTS region is just the impulse region in the original KZS. When $\zeta_{e} \sim \zeta_{s}, g R_{g}{ }^{-1 / v r_{g}}$ is a constant and Equation (5) gives $M \propto R_{g}{ }^{\beta / v r_{g}}$, which is consistent with the original KZS. Moreover, the FTS shows that the system in the impulse region does not cease to evolve. Instead, its evolution time scale is given by $\zeta_{e}$.

When $g_{0}$ is near the critical point, it has been shown that the driven dynamics of $M$ still exhibit scaling behaviors, which can be described by [38]

$$
M\left(g, R_{g}, g_{0}\right)=R_{g}{ }^{\beta / v r_{g}} f_{4}\left(g R_{g}{ }^{-1 / v r_{g}}, g_{0} R_{g}{ }^{-1 / v r_{g}}\right)
$$

where $g_{0}$ has been included in the scaling form. Since $g_{0}$ brings another time scale into the dynamics, i.e., the initial time scale $\zeta_{i} \sim g_{0}{ }^{-v z}$, which is larger than $\zeta_{e} \sim R_{g}{ }^{-z / r_{g}}$ for a small $g_{0}$, there is an initial crossover stage, bridging the region dominated by $\zeta_{i}$ and the FTS region. However, the critical slowing down makes this crossover stage macroscopically long. Thus, $g_{0}$ affects the driven dynamics during the whole process.

Another generalization of the KZS is that the thermal effects can be taken into account by including the temperature $T$ in the scaling theory [21,35-38]. However, it has been shown that for a closed quantum system, the status of temperature $T$ is closely related to the initial distance to the critical point [38]. To see the reason, one should at first note that for a closed system, the temperature involved in the driven dynamics can only be from the initial condition. Only when $g_{0}$ is near the critical point is the initial gap small compared with the temperature, and the number of thermal excitations can be macroscopically large enough to affect the following dynamics. Accordingly, the evolution of $M$ starting with a thermal state near the critical point satisfies [38]

$$
M\left(g, R_{g}, g_{0}, T\right)=R_{g} \beta / v r_{g} f_{5}\left(g R_{g}{ }^{-1 / v r_{g}}, g_{0} R_{g}{ }^{-1 / v r_{g}}, T R_{g}{ }^{-z / r_{g}}\right)
$$

\section{Effects Induced by the Initial Condition in the Kibble-Zurek Scaling for Changing the Symmetry-Breaking Field}

Besides the case of changing $g$, it has been shown that the dynamics of changing the symmetry-breaking field $h$ as $h=R_{h} t+h_{0}$ for a large $h_{0}$ also shows a scaling behavior $[22,23,28,29,34]$. In this case, $h_{0}$ is irrelevant. According to the FTS theory $[28,29,34]$, the effective time scale induced by the external driving is $\zeta_{e} \sim R_{h}{ }^{-z / r_{h}}$, in which $r_{h}=z+\beta \delta / v$, with $\delta$ being defined as $M \sim h^{1 / \delta}$ at $g=0$. 
Similar to the case of a small $g_{0}$, when $h_{0}$ is small, $h_{0}$ should also be included in the scaling theory. Accordingly, the order parameter satisfies

$$
M\left(h, R_{h}, h_{0}\right)=R_{h}^{\beta / v r_{h}} f_{6}\left(h R_{h}{ }^{-1 / v r_{h}}, h_{0} R_{h}{ }^{-1 / v r_{h}}\right)
$$

in which we have set $g=0$ for simplicity.

In Equation (8), when $h_{0}$ is large, the initial stage is an adiabatic stage. In this case, $h_{0}$ is irrelevant and Equation (8) restores the usual scaling form of FTS $[22,23,28]$. However, when $h_{0}$ is small, in the initial stage, the characterized time scale is $\zeta_{i} \sim h_{0}{ }^{-v z / \beta \delta}$, and $M \sim h_{0}{ }^{1 / \delta}$. Since $\zeta_{i} \gg \zeta_{e}$, the system will cross over to the FTS region. The cooperation between these crossover dynamics and the driven dynamics will make the dynamic behavior different from the usual KZS. For example, at the impulse-adiabatic boundary, at which $h R_{h}{ }^{-1 / v r_{h}}$ is a constant, $M$ depends on the driving rate $R_{h}$ and $h_{0}$. However, for the usual FTS, $M$ only depends on $R_{h}$.

When the initial state is an equilibrium state at finite $T$, the scaling theory should also include the temperature $T$,

$$
M\left(h, R_{h}, h_{0}, T\right)=R_{h}{ }^{\beta / v r_{h}} f_{7}\left(h R_{h}{ }^{-1 / v r_{h}}, h_{0} R_{h}{ }^{-1 / v r_{h}}, T R_{h}{ }^{-z / r_{h}}\right)
$$

Similar to the case of changing $g$ [38], here $T$ only has nontrivial effects when $h_{0}$ is small. When $h_{0}$ is large, the number of initial thermal excitations is tiny and thus has no effect on the following dynamics.

At $T=0$, the entanglement entropy $S$ also demonstrates scaling behaviors affected by the initial condition [40-44]. The entanglement entropy $S$ is defined as $S=-\operatorname{Tr}(\rho \log \rho)$, in which the base of the logarithm is 2 throughout and $\rho$ is the reduced density matrix of half of the system. For the 1D system near the critical point, the entanglement entropy scales as $S=(c / 6) \log \xi$, where $c$ is the central charge [40-44].

For $h=R_{h} t+h_{0}$, the scaling form of $\xi$ reads

$$
\xi\left(h, R_{h}, h_{0}\right)=R_{h}{ }^{-1 / r_{h}} f_{8}\left(h R_{h}{ }^{-1 / v r_{h}}, h_{0} R_{h}{ }^{-1 / v r_{h}}\right)
$$

Therefore, one can obtain the scaling form of the entanglement entropy $S$,

$$
S\left(h, R_{h}, h_{0}\right)=-\frac{c}{6 r_{h}} \log R_{h}+f_{9}\left(h R_{h}{ }^{-1 / v r_{h}}, h_{0} R_{h}{ }^{-1 / v r_{h}}\right)
$$

The scaling behavior of the entanglement entropy has been studied in the driven dynamics of the changing $g$ [45]. It has been shown that for a large $g_{0}$ and fixed $g R_{g}{ }^{-1 / v r_{g}}, S \sim-\left(c / 6 r_{g}\right) \log R_{g}$. Here, we demonstrate that similar scaling behavior also exists for changing the symmetry-breaking field.

\section{Model and Numerical Method}

The 1D quantum Ising model in a longitudinal field will be employed to illustrate the scaling theory. The Hamiltonian is [39]

$$
H=-\sum_{n} \sigma_{n}^{z} \sigma_{n+1}^{z}-w_{x} \sum_{n} \sigma_{n}^{x}-h \sum_{n} \sigma_{n}^{z}
$$

in which $\sigma_{n}^{z}$ and $\sigma_{n}^{x}$ are Pauli matrices in the $z$ and $x$ directions, respectively, at site $n ; w_{x}$ is the transverse field which is the fluctuation term; and $h$ is the longitudinal field which breaks the $Z_{2}$ symmetry. The order parameter $M$ is defined as $M \equiv<\sigma_{n}^{z}>$, where the angle brackets denote the expectation value of the operator at site $n$. The critical point for Equation (12) is $w_{x c}=1$ (and thus $\left.g \equiv w_{x}-1\right)$ and $h_{c}=0$. The exact critical exponents are $\beta=1, v=1, \delta=15, z=1$ [39], $r_{h}=23 / 8[28,29]$, and the central charge $c=1 / 2[40,41]$. In the following, we will consider the case for $g=0$.

We will employ the infinite time-evolving block decimation (iTEBD) algorithm [45] to simulate the driven dynamics. This algorithm is a variant of the time-dependent density-matrix renormalization 
group method [46-48]. For the zero temperature case, two steps must be implemented: first, calculating the ground state at $h_{0}$; second, calculating the evolution of $M$. To realize the first step, one should at first prepare a random state, and represent it in a matrix product state form via Vidal's decomposition [46], and then the ground state can be determined by imposing the imaginary time evolution operator on the matrix product state. The second step can be realized by a similar procedure: from the ground state, time dynamics can be calculated by imposing the evolution operator on the matrix product state, and at every time step, $h$ should be changed by a rate $R_{h}$.

For the driven dynamics starting from a thermal equilibrium state, we also need two steps. The first step is to calculate the thermal equilibrium state at $h_{0}$, i.e., $\rho_{0}=\exp \left[-H\left(h_{0}\right) / T\right] / Z$, in which $Z$ is the partition function. To do this, one should at first purify the identity matrix $I$ into a pure state $|\varphi\rangle_{I}$ by introducing an auxiliary system [47,48], which is maximally entangled to the physical system. After representing $|\varphi\rangle_{I}$ in the matrix product state, one can obtain a "thermal" pure state $|\varphi\rangle_{T} \equiv \exp [-H /(2 T)]|\varphi\rangle_{I}$ by imposing the imaginary time evolution operator on $|\varphi\rangle_{I}[47,48]$. The second step is to calculate the real time evolution state $|\varphi(t)\rangle_{T}$, which can be directly calculated by imposing the real time evolution operator on $|\varphi\rangle_{T}$. It has been shown that the dynamics of the order parameter $M$ can be directly calculated from $|\varphi(t)\rangle_{T}[47,48]$.

In the following calculations, the time interval is chosen to be 0.005 and 100 states are kept for both the zero temperature and finite temperature cases.

\section{Numerical Results}

We consider the driven dynamics of Equation (12) for changing the symmetry-breaking field $h$ as $h=R_{h} t+h_{0}$. For simplicity, we fix $g=0$, i.e., $w_{x}=1$. In Figure 1 , we compare the driven dynamics starting in the vicinity of the critical point and the driven dynamics starting far from the critical point. One finds that the curves for these two initial conditions are quite different. In Figure 1a, one finds that the hysteresis effect of $M$ for the evolution starting from the vicinity of the critical point is weaker than that for the evolution starting far from the critical point. In contrast, in Figure 1b, we find the hysteresis for the dynamics starting from the vicinity of the critical point is stronger than that for the dynamics starting far from the critical point.

To verify Equation (8), we plot $M$ versus $h$ for a small fixed $h_{0} R_{h}{ }^{-1 / v r_{h}}$ in Figure 2. After rescaling $M$ versus $h$ by $R_{h}$ in Figure 2b, we find that the rescaled curves collapse onto each other, verifying Equation (8). Therefore, we confirm that although the driven dynamics are affected by the initial condition, scaling behaviors also exist and the initial symmetry-breaking field $h_{0}$ should be included in the scaling form.
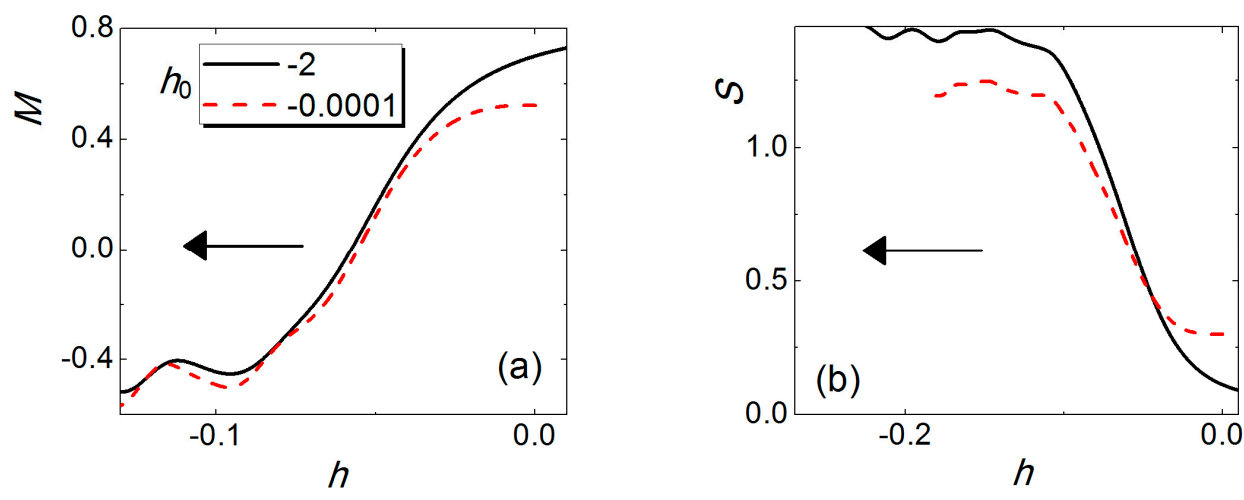

Figure 1. Comparison of the evolution of (a) the order parameter $M$ and (b) the entanglement entropy $S$ under decreasing $h$ with different initial conditions but the identical driving rate $R_{h}=0.009$. Arrows show the driven direction. 

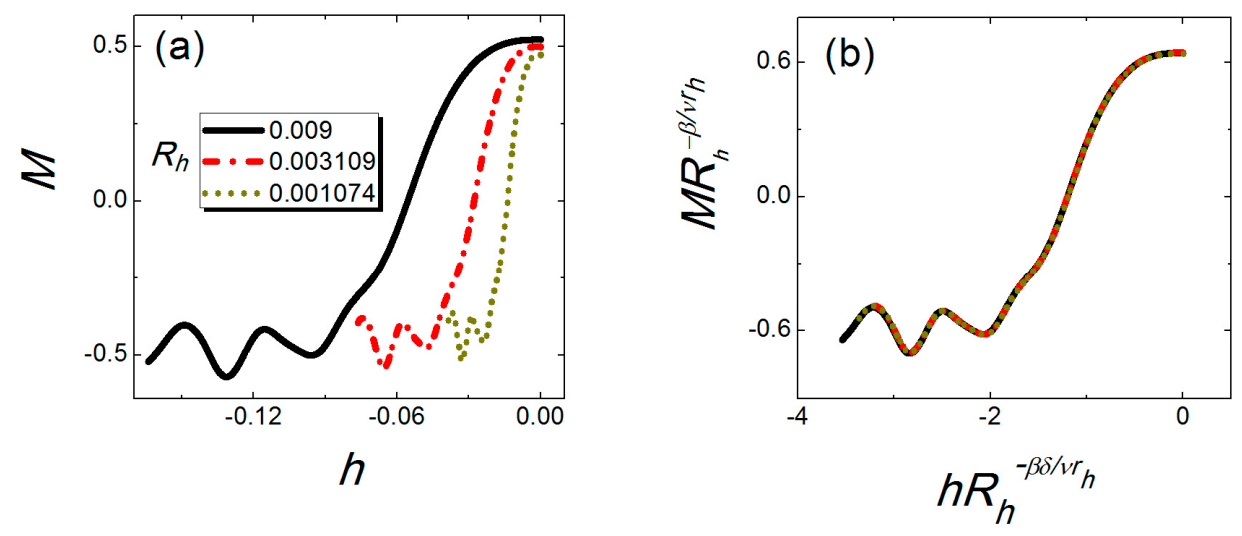

Figure 2. At $T=0$ the evolution of $M$ under decreasing $h$ with fixed $h_{0} R_{h}{ }^{-1 / v r_{h}}=0.002159$ for different three driving rates indicated. The curves before and after being rescaled are shown in (a) and (b), respectively.

The scaling theory including $h_{0}$ can also be verified according to the scaling behavior of the entanglement entropy $S$. According to Equation (11), we calculate $S+\left(c / 6 r_{h}\right) \log R_{h}$ for a small fixed $h_{0} R_{h}{ }^{-1 / v r_{h}}$. Figure 3 shows that the curves for different driving rates $R_{h}$ collapse onto each other after rescaling. These results confirm Equation (11).
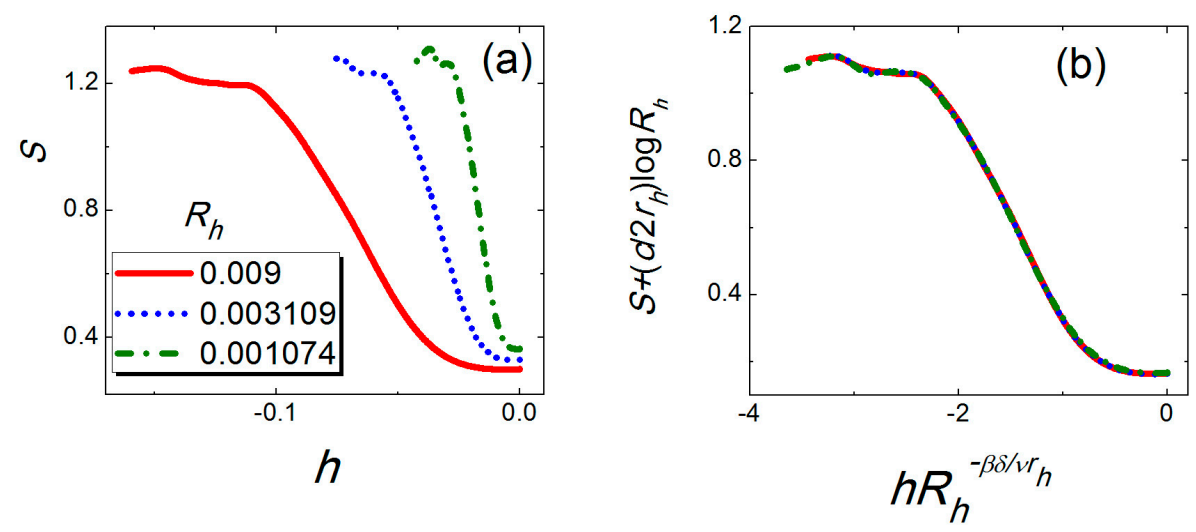

Figure 3. At $T=0$, the evolution of $S$ under decreasing $h$ with fixed $h_{0} R_{h}{ }^{-1 / v r_{h}}=0.002159$ for different three driving rates indicated is shown in (a). The curves for $S+\left(c / 6 r_{h}\right) \log R_{h}$ versus the rescaled $h$ are shown in (b).

Then, we verify the scaling theory at the finite temperature. For a small fixed $h_{0} R_{h}{ }^{-1 / v r_{h}}$ and a fixed $T R_{h}{ }^{-z / r_{h}}$, we find the evolution curves for different driving rates match each other after rescaling in Figure 4. This result confirms Equation (9). Since all the experiments are implemented at finite temperatures, this result also provides the experimental feasibility to examine the present scaling theory. 

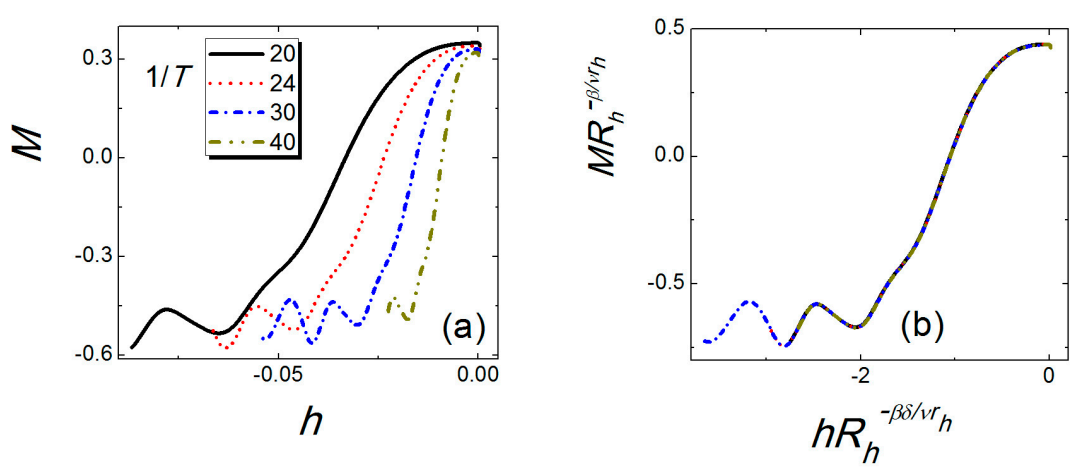

Figure 4. The evolution of $M$ under decreasing $h$ with fixed $h_{0} R_{h}{ }^{-1 / v r_{h}}=0.002159$ and $T R_{h}{ }^{-z / r_{h}}=1.583$ for four $T$ indicated. The curves before and after rescaling are shown in (a) and (b), respectively.

\section{Summary and Discussion}

In this paper, we have investigated the effects induced by the initial condition in the driven critical dynamics by changing the symmetry-breaking field. We have shown that when the initial symmetry-breaking field is small, the driven dynamics are quite different from the dynamics starting with a large symmetry-breaking field, which is required in the original finite-time scaling. In spite of this, we have demonstrated that the dynamics also exhibit scaling behaviors. By using the finite-time scaling theory, we obtain the scaling forms for the driven dynamics. In these scaling forms, the initial symmetry-breaking field should be included as an additional scaling variable. Furthermore, we also consider the finite temperature case. So this scaling theory can be examined in experiments. We have numerically verified the scaling theories by taking the 1D quantum Ising model as an example. We emphasize that for some experiments in which the usual Kibble-Zurek scaling cannot work, the present scaling theory may be a possible candidate to modify the scaling analysis. We also note that the Kibble-Zurek scaling has been generalized to the inhomogeneous cases, in which the relevant parameter depends on both the space and the time [49]. It has been shown that scaling behaviors in these systems are quite different from the original Kibble-Zurek mechanism [49]. So, it is appealing to explore the finite-time scaling and the initial effects therein.

Acknowledgments: Liang-Jun Zhai is supported by the National Natural Science Foundation of China (Grant No. 11547142); Shuai Yin is supported by the Ministry of Science and Technology (MOST) of Taiwan through Grant No. 104-2628-M-007-005-MY3.

Author Contributions: Shuai Yin conceived this subject; Shuai Yin and Liang-Jun Zhai performed the numerical simulation and analyzed the data; Shuai Yin and Liang-Jun Zhai wrote the paper. Both authors have read and approved the final manuscript.

Conflicts of Interest: The authors declare no conflict of interest.

\section{References}

1. Dziarmaga, J. Dynamics of a quantum phase transition and relaxation to a steady state. Adv. Phys. 2010, 59, 1063-1189. [CrossRef]

2. Polkovnikov, A.; Sengupta, K.; Silva, A.; Vengalattore, M. Colloquium: Nonequilibrium dynamics of closed interacting quantum systems. Rev. Mod. Phys. 2011, 83, 863-883. [CrossRef]

3. Dutta, A.; Aeppli, G.; Chakrabarti, B.K.; Divakaran, U.; Rosenbaum, T.F.; Sen, D. Quantum Phase Transitions in Transverse Field Spin Models: From Statistical Physics to Quantum Information, 1st ed.; Cambridge University Press: Cambridge, UK, 2015.

4. Kibble, T.W.B. Topology of cosmic domains and strings. J. Phys. A Math. Gen. 1976, 9, 1387-1398. [CrossRef]

5. Zurek, W.H. Cosmological experiments in superfluid helium? Nature 1985, 317, 505-508. [CrossRef]

6. Zurek, W.H.; Dorner, U.; Zoller, P. Dynamics of a Quantum Phase Transition. Phys. Rev. Lett. 2005, 95, 105701. [CrossRef] [PubMed] 
7. Dziarmaga, J. Dynamics of a Quantum Phase Transition: Exact Solution of the Quantum Ising Model. Phys. Rev. Lett. 2005, 95, 245701. [CrossRef] [PubMed]

8. Polkovnikov, A. Universal adiabatic dynamics in the vicinity of a quantum critical point. Phys. Rev. B 2005, 72, 161201. [CrossRef]

9. Damski, B.; Zurek, W.H. Dynamics of a Quantum Phase Transition in a Ferromagnetic Bose-Einstein Condensate. Phys. Rev. Lett. 2007, 99, 130402. [CrossRef] [PubMed]

10. Sen, D.; Sengupta, K.; Mondal, S. Defect Production in Nonlinear Quench across a Quantum Critical Point. Phys. Rev. Lett. 2008, 101, 016806. [CrossRef] [PubMed]

11. Deng, S.; Ortiz, G.; Viola, L. Dynamical non-ergodic scaling in continuous finite-order quantum phase transitions. Europhys. Lett. 2008, 84, 67008. [CrossRef]

12. Damski, B.; Zurek, W.H. Soliton Creation during a Bose-Einstein Condensation. Phys. Rev. Lett. 2010, 104, 160404. [CrossRef] [PubMed]

13. Francuz, A.; Dziarmaga, J.; Gardas, B.; Zurek, W.H. Space and time renormalization in phase transition dynamics. Phys. Rev. B 2016, 93, 075134. [CrossRef]

14. Antunes, N.D.; Gandra, P.; Rivers, R.J. Is domain formation decided before or after the transition? Phys. Rev. D 2006, 73, 125003. [CrossRef]

15. Damski, B.; Zurek, W.H. Adiabatic-impulse approximation for avoided level crossings: From phase-transition dynamics to Landau-Zener evolutions and back again. Phys. Rev. A 2006, 73, 063405. [CrossRef]

16. Del Campo, A.; Zurek, W.H. Universality of phase transition dynamics: Topological defects from symmetry breaking. Int. J. Mod. Phys. A 2014, 29, 1430018. [CrossRef]

17. Deutschländer, S.; Dillmann, P.; Maret, G.; Keim, P. Kibble-Zurek mechanism in colloidal monolayers. Proc. Natl. Acad. Sci. USA 2015, 112, 6925-6930. [CrossRef] [PubMed]

18. De Grandi, C.; Polkovnikov, A.; Sandvik, A.W. Universal nonequilibrium quantum dynamics in imaginary time. Phys. Rev. B 2011, 84, 224303. [CrossRef]

19. Kolodrubetz, M.; Clark, B.K.; Huse, D.A. Nonequilibrium Dynamic Critical Scaling of the Quantum Ising Chain. Phys. Rev. Lett. 2012, 109, 015701. [CrossRef] [PubMed]

20. Kolodrubetz, M.; Pekker, D.; Clark, B.K.; Sengupta, K. Nonequilibrium dynamics of bosonic Mott insulators in an electric field. Phys. Rev. B 2012, 85, 100505. [CrossRef]

21. Chandran, A.; Erez, A.; Gubser, S.S.; Sondhi, S.L. Kibble-Zurek problem: Universality and the scaling limit. Phys. Rev. B 2012, 86, 064304. [CrossRef]

22. Yin, S.; Qin, X.; Lee, C.; Zhong, F. Finite-time scaling of dynamic quantum criticality. arXiv 2012, arXiv:1207.1602.

23. Yin, S.; Mai, P.; Zhong, F. Nonequilibrium quantum criticality in open systems: The dissipation rate as an additional indispensable scaling variable. Phys. Rev. B 2014, 89, 094108. [CrossRef]

24. Ulm, S.; Roßnagel, J.; Jacob, G.; Degünther, C.; Dawkins, S.; Poschinger, U.; Nigmatullin, R.; Retzker, A.; Plenio, M.; Schmidt-Kaler, F.; et al. Observation of the Kibble-Zurek scaling law for defect formation in ion crystals. Nat. Commun. 2013, 4, 2290. [CrossRef] [PubMed]

25. Pyka, K.; Keller, J.; Partner, H.L.; Nigmatullin, R.; Burgermeister, T.; Meier, D.M.; Kuhlmann, K.; Retzker, A.; Plenio, M.B.; Zurek, W.H.; et al. Topological defect formation and spontaneous symmetry breaking in ion Coulomb crystals. Nat. Commun. 2013, 4, 2291. [CrossRef] [PubMed]

26. Navon, N.; Gaunt, A.L.; Smith, R.P.; Hadzibabic, Z. Critical dynamics of spontaneous symmetry breaking in a homogeneous Bose gas. Science 2015, 347, 167-170. [CrossRef] [PubMed]

27. Clark, L.W.; Feng, L.; Chin, C. Universal space-time scaling symmetry in the dynamics of bosons across a quantum phase transition. Science 2016, 354, 606-610. [CrossRef] [PubMed]

28. Gong, S.; Zhong, F.; Huang, X.; Fan, S. Finite-time scaling via linear driving. New J. Phys. 2010, $12,043036$. [CrossRef]

29. Zhong, F. Finite-time Scaling and its Applications to Continuous Phase Transitions. In Applications of Monte Carlo Method in Science and Engineering; Mordechai, S., Ed.; InTech: Rijeka, Croatia, 2011.

30. Liu, C.W.; Polkovnikov, A.; Sandvik, A.W. Quantum versus Classical Annealing: Insights from Scaling Theory and Results for Spin Glasses on 3-Regular Graphs. Phys. Rev. Lett. 2015, 114, 147203. [CrossRef] [PubMed]

31. Liu, C.W.; Polkovnikov, A.; Sandvik, A.W.; Young, A.P. Universal dynamic scaling in three-dimensional Ising spin glasses. Phys. Rev. E 2015, 92, 022128. [CrossRef] [PubMed] 
32. Hu, Q.; Yin, S.; Zhong, F. Scaling of the entanglement spectrum in driven critical dynamics. Phys. Rev. B 2015, 91, 184109. [CrossRef]

33. Huang, Y.; Yin, S.; Hu, Q.; Zhong, F. Kibble-Zurek mechanism beyond adiabaticity: Finite-time scaling with critical initial slip. Phys. Rev. B 2016, 93, 024103. [CrossRef]

34. Feng, B.; Yin, S.; Zhong, F. Theory of driven nonequilibrium critical phenomena. Phys. Rev. B 2016, 94, 144103. [CrossRef]

35. De Grandi, C.; Gritsev, V.; Polkovnikov, A. Quench dynamics near a quantum critical point. Phys. Rev. B 2010, 81, 012303. [CrossRef]

36. De Grandi, C.; Gritsev, V.; Polkovnikov, A. Quench dynamics near a quantum critical point: Application to the sine-Gordon model. Phys. Rev. B 2010, 81, 224301. [CrossRef]

37. Deng, S.; Ortiz, G.; Viola, L. Dynamical critical scaling and effective thermalization in quantum quenches: Role of the initial state. Phys. Rev. B 2011, 83, 094304. [CrossRef]

38. Yin, S.; Lo, C.-Y.; Chen, P. Scaling in driven dynamics starting in the vicinity of a quantum critical point. Phys. Rev. B 2016, 94, 064302. [CrossRef]

39. Sachdev, S. Quantum Phase Transitions; Cambridge University Press: Cambridge, UK, 1999.

40. Eisert, J.; Cramer, M.; Plenio, M. Colloquium: Area laws for the entanglement entropy. Rev. Mod. Phys. 2010, 82, 277-306. [CrossRef]

41. Amico, L.; Fazio, R.; Osterloh, A.; Vedral, V. Entanglement in many-body systems. Rev. Mod. Phys. 2008, 80, 517-576. [CrossRef]

42. Osterloh, A.; Amico, L.; Falci, G.; Fazio, R. Scaling of entanglement close to a quantum phase transition. Nature 2002, 416, 608-610. [CrossRef] [PubMed]

43. Laflorencie, N. Quantum entanglement in condensed matter systems. Phys. Rep. 2016, 646, 1-59. [CrossRef]

44. Calabrese, P.; Cardy, J. Evolution of entanglement entropy in one-dimensional systems. J. Stat. Mech. 2005, 2005, 04010. [CrossRef]

45. Cincio, L.; Dziarmaga, J.; Rams, M.; Zurek, W. Entropy of entanglement and correlations induced by a quench: Dynamics of a quantum phase transition in the quantum Ising model. Phys. Rev. A 2007, 75, 052321. [CrossRef]

46. Vidal, G. Classical Simulation of Infinite-Size Quantum Lattice Systems in One Spatial Dimension. Phys. Rev. Lett. 2007, 98, 070201. [CrossRef] [PubMed]

47. Verstraete, F.; García-Ripoll, J.J.; Cirac, J.I. Matrix Product Density Operators: Simulation of Finite-Temperature and Dissipative Systems. Phys. Rev. Lett. 2004, 93, 207204. [CrossRef] [PubMed]

48. Schollwöck, U. The density-matrix renormalization group in the age of matrix product states. Ann. Phys. 2011, 326, 96-192. [CrossRef]

49. Del Campo, A.; Retzker, A.; Plenio, M.B. Inhomogeneous Kibble-Zurek mechanism: Vortex nucleation during Bose-Einstein condensation. New J. Phys. 2011, 13, 083022. [CrossRef]

(C) 2016 by the authors; licensee MDPI, Basel, Switzerland. This article is an open access article distributed under the terms and conditions of the Creative Commons Attribution (CC-BY) license (http://creativecommons.org/licenses/by/4.0/). 